\title{
Conhecimentos Nutricionais e Perfil Alimentar: Discussão dos Saberes e Práticas de Acadêmicos de Pedagogia
}

\section{Nutritional Knowledge and Dietary Profile: Understanding and Practices Discussion from Pedagogy Students}

\author{
Camilla Pastore Anizelli; ; Marcela Komechen Brecailo ${ }^{\text {b*; }}$ Caryna Eurich Mazur

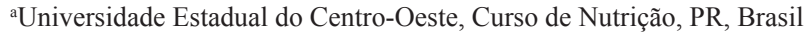 \\ ${ }^{b}$ Universidade Estadual do Centro-Oeste, Departamento de Nutrição, PR, Brasil \\ 'Universidade Federal do Paraná, Programa de Pós-Graduação em Segurança Alimentar e Nutricional, PR, Brasil \\ *E-mail: marbrecailo@gmail.com \\ Recebido: 21 de janeiro de 2014; Aceito: 20 de maio de 2014
}

\begin{abstract}
Resumo
Nos últimos anos, vem crescendo a incidência de doenças crônicas não-transmissíveis entre a população, devido aos maus hábitos alimentares e a falta de conhecimento ou aplicação das práticas saudáveis da alimentação. Sendo assim, o conhecimento nutricional se faz necessário para que novos conceitos sejam criados e implantados na alimentação cotidiana. A presente pesquisa é um estudo transversal analítico, cujo grupo foi composto por 99 universitários do curso de Pedagogia de Guarapuava - Paraná. A avaliação foi feita por meio de questionário validado com 25 possíveis acertos, e os hábitos alimentares foram identificados com o auxílio de um recordatório de 24 horas. O número médio de acertos dos alunos pesquisados foi de 63,92\%, demonstrando baixo conhecimento em temas específicos de nutrição. Os hábitos alimentares se caracterizaram por um baixo número de refeições/dia. O consumo de leite e derivados, hortaliças e frutas foi abaixo da recomendação diária, sendo que estes hábitos devem ser implementados no cotidiano dos avaliados. Não houve correlação entre o número de acertos no questionário e o consumo de frutas, hortaliças e leite e derivados. Portanto, além da necessidade de incorporar a educação alimentar e nutricional com a prática pedagógica, constituindo uma apta formação de pedagogos, é fundamental um maior entendimento da subjetividade relacionada ao consumo alimentar.
\end{abstract}

Palavras-chave: Estudantes. Educação Infantil. Educação Alimentar e Nutricional.

\begin{abstract}
In recent years, the grown incidence of non-transmissible chronic diseases (NTCDs) among the general population is due to poor eating habits and lack of knowledge or practical application of healthy consumption. Thus, the nutritional knowledge is necessary so that new concepts are created and deployed in daily diet. This was a cross-sectional analytical study, which engaged 99 University Pedagogy course students from Guarapuava - Paraná, Brazil. The evaluation was done by a validated questionnaire with 25 possible arrangements for correct answering; and food intake was identified with a 24-hour recall. The mean number of correct answers was of 15.98 ( \pm 3.074$)$, demonstrating little knowledge on specific topics of nutrition. Dietary habits were characterized by a low number of daily meals. Milk, fruit and vegetables intakes were below the daily recommended portions, showing that these habits must be implemented in everyday routine. A greater number of correct answers from nutrition knowledge questionnaire were not correlated to a higher consumption of fruits, vegetables and milk. Therefore, besides the need for nutrition education with pedagogical practice constituting a suitable training of pedagogues, it is essential to think about the subjectivity that leads to consumption.
\end{abstract}

Keywords: Students. Child Rearing. Food and Nutrition Education.

\section{Introdução}

A mudança dos hábitos alimentares ocorrida nas últimas décadas no Brasil está entre as principais causas do aumento das doenças crônicas não-transmissíveis - DCNT em grande parte da população ${ }^{1}$. Neste âmbito, estudos epidemiológicos de base populacional e ensaios clínicos randomizados levaram a Organização Mundial da Saúde - OMS a considerar o importante papel da dieta na prevenção e controle de morbimortalidades ${ }^{2}$.

Para que a população faça o uso desse benefício, é necessário que se tenha conhecimento das práticas saudáveis em alimentação. $O$ conhecimento nutricional pode ser estabelecido como o processo cognitivo individual, relativo à informação sobre alimentação e nutrição, ou ainda, é o que se sabe sobre os alimentos e alimentação ${ }^{3}$.

A alimentação, desde os primeiros meses de vida, é essencial à socialização da criança e à sua inclusão na cultura de seu povo, pois no momento da alimentação, a criança entra em contato com um sistema de representações e práticas de sua cultura ${ }^{4}$. Essa é a fase em que a criança se encontra em crescimento e desenvolvimento, criando a capacidade de aprender e se adaptar aos estímulos recebidos. Dessa maneira, torna-se essencial, para uma boa formação, que ela cresça num ambiente incentivador, capaz de desenvolver suas habilidades físicas, motoras, psíquicas e sociais ${ }^{5}$.

De acordo com Veiga ${ }^{6}$, todo projeto político-pedagógico PPP deve ser construído e vivenciado por todos os envolvidos com o processo educativo, buscando a organização do trabalho 
pedagógico na escola. Então, no momento da alimentação, deve ser estudado como a educação alimentar e nutricional será trabalhada, pois deve seguir as necessidades de cada população. Portanto, é preciso tempo para acompanhar e avaliar a ação desta educação.

O vínculo alimentar da criança se inicia já no seio da mãe. Em seguida, começa a receber outros alimentos consumidos pela família. Logo após, é inserida nas instituições de ensino e educação, onde aprende e adquire novos hábitos, contribuindo, assim, para a formação de um hábito alimentar. Portanto, é perceptível que, de acordo com a contribuição teórica de Bourdieu ${ }^{7}$, o hábito alimentar seja formado no contexto em que a criança está envolvida, desde o ambiente familiar ao escolar. E, segundo Marinho et al. ${ }^{8}$, esse processo compreende aspectos sociodemográficos, escolaridade, renda e local de moradia, além dos aspectos subjetivos e culturais envolvidos nesse processo. Porém, sabe-se que a criança é disseminadora dos conhecimentos adquiridos ${ }^{9}$ e transfere para sua família os conhecimentos em saúde e nutrição abordados na escola.

Portanto, mostra-se necessário o conhecimento dos profissionais da área de pedagogia em relação à nutrição, para disseminação deste conhecimento às crianças e suas famílias, contribuindo com a promoção da saúde para toda a população. Deste modo, o objetivo deste trabalho foi avaliar o perfil alimentar e os conhecimentos nutricionais de universitários do curso de Pedagogia, discutindo sua influência na própria alimentação e na construção de saberes em nutrição dos futuros alunos.

\section{Material e Métodos}

Tratou-se de um estudo transversal analítico, cujo grupo foi composto por 99 universitários, de uma universidade estadual de Guarapuava, no estado do Paraná. A população do estudo foi constituída por acadêmicos de 19 a 59 anos, pertencentes às segundas e terceiras séries do curso de Pedagogia, diurnas e noturnas, devidamente matriculados na Instituição de Ensino Superior, e que concordaram voluntariamente em participar do estudo, assinando um Termo de Consentimento Livre Esclarecido (TCLE).

Esse estudo foi aprovado pelo Comitê de Ética em Pesquisa - COMEP da UNICENTRO, sob número de parecer 241/2011 e a coleta de dados só se iniciou após a aprovação do Comitê. O período de coleta de dados compreendeu os meses de abril e maio de 2012.

Para a avaliação do estado nutricional, foi realizada uma coleta de dados antropométricos autorrelatados, em que os próprios acadêmicos responderam seu peso $(\mathrm{Kg})$ e altura $(\mathrm{m})$. O Índice de Massa Corporal - IMC foi calculado considerandose a razão peso atual $(\mathrm{kg})$ e o quadrado da estatura $\left(\mathrm{m}^{2}\right)$. Estudos ${ }^{10}$ demonstram que o peso e a altura referidos possuem alta concordância com os dados obtidos diretamente, sendo uma alternativa válida quando não se consegue esses dados por outros métodos. Para classificação e diagnóstico do estado nutricional segundo o IMC, foram utilizados os pontos de corte para adultos propostos pela World Health Organization ${ }^{11}$.

O consumo alimentar foi identificado a partir de um recordatório de 24 horas $(\mathrm{R} 24 \mathrm{~h})^{12}$, de modo que cada participante do estudo listou todos os alimentos consumidos no dia anterior ao inquérito, e analisado de acordo com número de porções de importantes grupos alimentares que observam baixo consumo em diversas populações ${ }^{1}$.

Para a avaliação dos conhecimentos nutricionais, foi utilizado o questionário validado por Guadagnin ${ }^{13}$, com 14 questões e posssibilidade de acerto de 25 pontos. As questões versam sobre tópicos gerais da alimentação e assuntos específicos de nutrição, como presença de sódio, açúcar, gorduras nos alimentos e suas consequências.

Os dados do estudo foram tabulados com o auxílio do programa Excel $^{\circledR} 2003$ e a análise estatística foi realizada por meio de frequências, médias, desvio-padrão (estatística descritiva) e o coeficiente de correlação de Pearson para verificar a relação entre conhecimento teórico e prática alimentar. Para as análises foi utilizado o software SPSS $^{\circledR \text { vers16.0. }}$

\section{Resultados e Discussão}

A população estudada foi constituída de 99 estudantes universitários que responderam o questionário nutricional, de ambos os sexos, sendo $91,9 \%(\mathrm{n}=91)$ do sexo feminino, $6,1 \%$ $(n=6,1)$ do sexo masculino e $2,0 \%(n=2)$ não completaram esta informação. Com relação à idade, obteve-se média de $24,45( \pm 6$,$) anos, sendo 33,0 \%$ da população jovem adulto e o restante de $67,0 \%$ adultos.

Considerando que $5,1 \%(n=5)$ do total $(n=99)$ dos universitários não respondeu seu peso e a sua altura, o estado nutricional da população estudada encontra-se na Tabela 1.

Tabela 1: Estado nutricional de universitários ( $\mathrm{n}=94)$, Guarapuava, 2012.

\begin{tabular}{lccc}
\hline & M $( \pm \mathbf{D P})$ & $\mathbf{\%}$ & $\mathbf{n}$ \\
\hline IMC & $22,91( \pm 4,70)$ & - & - \\
Magreza & - & 9,6 & 9 \\
Eutrofia & - & 66,0 & 62 \\
Sobrepeso & - & 17,0 & 16 \\
Obesidade & - & 7,4 & 7 \\
Total & - & 100,0 & 94 \\
\hline
\end{tabular}

Fonte: Dados da pesquisa.

$\mathrm{Na}$ investigação sobre conhecimentos nutricionais, de um total de possibilidade de 25 acertos, o número médio de acertos dos alunos pesquisados obteve média de 15,98( $\pm 3,074)$.

Ao serem questionados sobre a influência dos alimentos na saúde, 98\% ( $\mathrm{n}=97)$ dos entrevistados concordaram que consumindo determinados tipos de alimentos reduzem-se as chances de desenvolvimento de doenças. Em relação aos bons hábitos que reduzem as chances de doenças, 98\% dos avaliados (n=97) assinalaram a opção em que uma alimentação 
com mais fibras, mais frutas e hortaliças, menos gordura e uma mudança no consumo de outros alimentos/nutrientes (por exemplo, sal e açúcar), podem reduzir as chances do aparecimento de doenças.

Ainda com relação aos acertos, questionados sobre se as frutas, os legumes e as verduras são essenciais para o bom funcionamento intestinal, 77,8\% $(n=77)$ dos avaliados marcaram a opção "certo". Quando solicitados para definir que alimento continha maior quantidade de gordura, entre "1 pedaço de bolo" ou "1 fatia de pão integral", a maioria dos entrevistados, ou seja, 83,8\% $(n=83)$, marcaram a alternativa um pedaço de bolo, sendo esta a alternativa correta.

$\mathrm{Na}$ pergunta referente à quantidade de gordura trans, a opção biscoito recheado obteve um percentual de $81,8 \%$ $(\mathrm{n}=81)$, em que a maioria dos universitários considerou que ele é um alimento que possui alta quantidade dessa gordura. Ao serem questionados se os vegetais cozidos tinham pouco ou muito sal, 78,8\% $(n=78)$ dos entrevistados afirmaram que eles possuem pouco sal na sua composição. Ainda nessa questão, as opções macarrão instantâneo, tempero industrializado e orégano obtiveram percentuais de $74,7 \%(n=74) ; 93,9 \%$ $(n=93)$ e $85,9 \%(n=85)$, respectivamente, considerando-os como alimentos que contêm muito sal. Observa-se que o tempero natural orégano é tido como um alimento rico em sódio para a maior parte desta população, sendo este um conceito errôneo.

Ao serem perguntados se o feijão com arroz é uma fonte completa de proteínas e rica em fibras, 74\% $(n=74,7)$ dos universitários concordaram com essa afirmação. $\mathrm{O}$ arroz com feijão (na proporção aproximada de 2:1) é uma combinação altamente nutritiva, e é o prato típico brasileiro. No entanto, nos últimos tempos observou-se uma diminuição no seu consumo e aumento considerável no consumo de carnes ${ }^{14,15}$.

Diante dos erros mais prevalentes nas respostas dos universitários, em relação à na pergunta que questionava sobre o porcionamento correto de frutas, 52,5\% ( $\mathrm{n}=52)$ não sabiam a sua correta recomendação diária, assim como aconteceu nas porções de hortaliças, em que 93\% $(n=93,9)$ dos avaliados também não conheciam a quantidade correta de consumo diário.

Ao serem questionados sobre quais eram os alimentos que possuíam gordura trans, as opções ovo, óleo de soja e azeite de oliva obtiveram as maiores taxas de erro, sendo eles: $51,5 \%$ $(n=51) ; 77,8 \%(n=77)$ e $52,5 \%(n=52)$ respectivamente. Por fim, ao serem questionados sobre qual era uma opção de lanche não saudável, 52,5\% $(\mathrm{n}=52)$ assinalaram opções erradas.

Analisando o Recordatório de 24 horas (R24h), preenchido pelos 99 universitários avaliados, percebeu-se que o número total de refeições diárias foi em média de 4,49 $( \pm 1,155)$, sendo que o mínimo de refeições realizadas diariamente foi de uma e o máximo de oito.

A média do consumo de frutas foi de 0,52 $( \pm 0,747)$ porções diárias, com quantidade mínima de zero e a máxima de três porções por dia. Para as hortaliças, a média obtida foi de 0,68 $( \pm 0,754)$ porções diárias, com um número mínimo de zero e o máximo de três ao dia. Com relação à quantidade de leite e derivados, obteve-se a média de $0,98( \pm 0,903)$ porções diárias, sendo a quantidade mínima de zero e a máxima de três durante o dia. Os outros grupos alimentares não foram avaliados nessa pesquisa.

Em relação ao corolário de um maior conhecimento nutricional, objetivou-se correlacioná-lo ao estado nutricional, evidenciado pelo IMC, e ao consumo alimentar. Os resultados estão descritos na Tabela 2.

Tabela 2: Correlação entre número de acertos em conhecimentos nutricionais e práticas alimentares adequadas, Guarapuava, 2012.

\begin{tabular}{lcc}
\hline & \multicolumn{2}{c}{$\begin{array}{c}\text { Número total } \\
\text { de acertos }\end{array}$} \\
\cline { 2 - 3 } & $\mathbf{r}$ & $\mathbf{p}$ \\
\hline $\mathrm{IMC}\left(\mathrm{kg} / \mathrm{m}^{2}\right)$ & 0,032 & 0,763 \\
Número total de refeições & 0,080 & 0,429 \\
Consumo diário de porções de frutas & 0,147 & 0,147 \\
Consumo diário de porções de hortaliças & $-0,091$ & 0,371 \\
Consumo diário de porções de leite e derivados & 0,154 & 0,128 \\
\hline
\end{tabular}

Fonte: Dados da pesquisa.

Sobre a faixa etária estudada nesta pesquisa e a promoção de uma alimentação saudável, dois aspectos podem ser considerados: a mudança de um comportamento alimentar em longo prazo é um objetivo com elevadas taxas de insucesso; e os hábitos alimentares da idade jovem e adulta estão relacionados com os aprendidos na infância ${ }^{16}$. Com isso, percebe-se que, embora a população seja composta por adultos e adolescentes, ambos grupos possuem hábitos alimentares similares, talvez pela proximidade de estilo de vida, influência das amizades ou devido ao pouco tempo para preparar as suas refeições. Uma vez que ainda são estudantes, o fator econômico também pode ser uma grande influência nas escolhas alimentares.

Com relação ao estado nutricional, percebe-se, na população, alta prevalência de magreza $(9,6 \%)$ e também de excesso de peso $(24,4 \%)$. A transição nutricional é um fenômeno reconhecido no Brasil das últimas décadas. Após os anos 1990, a prevalência de desnutrição e de excesso de peso alterou-se de forma alarmante. $O$ déficit de peso na população brasileira adulta é de $2,7 \%$. Este fato não é corroborado pelo presente estudo, em que se encontrou 3,56 vezes mais magreza (9,6\%). Em contraposição, a taxa de excesso de peso foi $50,2 \%$ menor do que na população em geral: $24,4 \%$ na população estudada, comparando-se a $49 \%$ na população brasileira adulta ${ }^{17,18}$. Constata-se que a transição nutricional até hoje não afeta os diferentes grupos populacionais da mesma forma, exigindo aconselhamento nutricional específico para esta população, em suas dificuldades em manter um peso saudável.

Nos resultados das questões respondidas pelos universitários que abordavam sobre algumas práticas 
alimentares saudáveis, foram encontrados os maiores números de acertos, assim como observado no estudo de Silva et al. ${ }^{19}$. Acredita-se que isso tenha acontecido porque estas informações encontram-se mais divulgadas pela mídia. Porém, abordando-se temas específicos como gorduras trans, gorduras saturadas, sódio e opção saudável, não foi identificado esse conhecimento na população estudada, o que pode ser um fator contribuinte para erros alimentares e até mesmo formação de opiniões errôneas ou incompletas em seu futuro público-alvo, os alunos.

Segundo Dalla Costa et al. ${ }^{15}$, os hábitos alimentares se modificam muito rapidamente. A mídia os constrói e os substitui. Essa influência pode ser verificada em seu estudo desenvolvido no Brasil, o qual mostrou que a propaganda recebe lugar de destaque como fonte de informação sobre questões nutricionais, entre adolescentes e jovens.

Porém, deve-se ter cuidado com as informações repassadas pela mídia, pois elas tendem ao sensacionalismo, trazendo informações nem sempre científicas, o que pode ter contribuído para a alta taxa de erro em algumas perguntas no questionário realizado pela presente pesquisa ${ }^{20}$.

No quesito número de refeições, os resultados desta pesquisa foram desfavoráveis, sendo que parte dos pesquisados mantinham grandes intervalos de tempo sem realizar algum tipo de refeição: 51 entrevistados $(51,5 \%)$ relataram realizar menos de quatro refeições diárias, sendo esta uma atitude negativa de hábito alimentar. Demonstrase a necessidade de explicações quanto a prática de 5 a 6 refeições ao dia, sendo importante não pular refeições, o que é prejudicial à saúde e ao bem-estar físico ${ }^{21}$.

Apesar de terem apresentado conhecimento em alguns temas básicos em relação à alimentação saudável, a pesquisa mostra que os participantes não vinculavam o conhecimento com as práticas adotadas no cotidiano.

Estudos transversais que utilizaram questionários de frequência alimentar encontraram baixa ingestão de frutas e hortaliças. Menos de $10 \%$ da população adulta atinge as recomendações de consumo de frutas, verduras e legumes, em que os homens apresentaram menores frequências de consumo de todas as verduras, de grande parte dos legumes e das frutas ${ }^{17}$. Em estudo realizado pelo Instituto Nacional do Câncer - INCA, quase metade (45\%) dos jovens de 15 a 24 anos do município de São Paulo consumiam frutas, legumes ou verduras menos que cinco vezes por semana ${ }^{22}$, o que se assemelha ao presente estudo.

A promoção do consumo de frutas e hortaliças é uma prioridade mundial para a melhoria da saúde da população. É conhecido o efeito protetor das frutas e hortaliças para DCNT e na manutenção do peso corporal. Nesse sentido, a OMS recomenda o consumo diário de $400 \mathrm{~g}$ de frutas para a prevenção destas doenças. Também é recomendado verificar se o consumo regular de frutas e hortaliças é de, no mínimo, cinco dias na semana, o que seria um marcador de alimentação saudável ${ }^{23}$.
O consumo médio per capita também mostra grandes variações entre as Grandes Regiões, com arroz, feijão, carne bovina e leite integral sendo mais consumidos na Região Centro-Oeste. O consumo de iogurtes se destaca nas Regiões Sudeste e Sul. Na Região Norte, as preparações à base de leite, que incluem os mingaus, foram mais consumidas do que nas outras regiões, sendo que as Regiões Sul e Centro-Oeste apresentaram valores médios per capita muito baixos ${ }^{17}$.

Monteiro et $a l .{ }^{24}$ realizaram uma investigação nutricional entre os anos de 1988 e 1986, e encontraram um importante aumento na oferta de carnes, de leite e seus derivados, porém houve reduções importantes no consumo de óleos e de gorduras vegetais, o que não condiz com a realidade dos dias de hoje.

Em trabalho anterior ${ }^{25}$ sobre a evolução do consumo alimentar nas áreas metropolitanas brasileiras entre as décadas de 60 e 80, foram constatadas duas tendências: a expansão no consumo relativo de produtos de origem animal (sobretudo leite e derivados, mas também carnes e ovos) em detrimento de cereais, leguminosas e raízes e tubérculos; e a substituição intensa de gorduras animais (banha, toucinho e manteiga) por óleos vegetais e margarinas. Um aspecto negativo das mudanças foi o aumento exagerado no conteúdo lipídico total da dieta, em que, em 1988, a proporção de calorias lipídicas atingiu cerca de $30 \%$ da dieta, ou o limite máximo recomendado para esse indicador. Atualmente, a situação não mostra-se melhor, sendo que a população adulta brasileira consegue, em média, $27 \%$ das calorias totais da dieta em lipídios e o consumo de coleterol varia entre 186,3 e 282,1 mg diariamente ${ }^{17}$.

Segundo os dados de correlação analisada na Tabela 2, observa-se que um maior conhecimento sobre as questões teóricas em alimentação e nutrição não levou necessariamente a uma prática alimentar mais adequada, também não influenciando o estado nutricional desta população. Este fato evidencia que o conhecimento nutricional foi insuficiente para atuar sobre o comportamento alimentar, em virtude das inúmeras influências que as escolhas sofrem, de ordem social, econômica, familiar e psicológica ${ }^{3}$.

Silva et $a l .{ }^{19}$, em seu estudo sobre conhecimentos nutricionais por crianças, observaram que professores de Ensino Infantil possuem um conhecimento sobre Nutrição Infantil, abrangendo, por exemplo, introdução de frutas e verduras na alimentação, porém deixam em segundo plano as atividades pedagógicas deste tema, as quais poderiam ser realizadas em um programa de educação nutricional, construído coletivamente e inserido no projeto políticopedagógico da escola.

Para que o professor se transforme em agente promotor de hábitos alimentares saudáveis é essencial que ele possua, além do conhecimento dos preceitos teóricos de dieta equilibrada, uma postura consciente de sua atuação na formação dos hábitos alimentares da criança ${ }^{26}$.

Os hábitos alimentares da população escolar estão 
determinados por uma série de fatores históricos, sociais, religiosos, econômicos e geográficos inerentes a cada comunidade. Oliveira e Thébaud-Mony ${ }^{27}$ assinalam que a alimentação é um fato social. Dessa forma, a escola é um ambiente propício para a aplicação de programas de educação em saúde e a formação de hábitos saudáveis, pois ela está inserida em todas as dimensões do aprendizado: ensino, relações lar-escola-comunidade e ambiente físico e emocional.

Um programa de capacitação, em relação à educação nutricional, deveria ser instituído para que estes profissionais pudessem atender as necessidades das crianças e adolescentes e transmitir informações corretas sobre conceitos nutricionais, visando à mudança de concepção e atitude. Porém, urge a necessidade de repensar a educação nutricional como construtora de conhecimento e geradora de ações, uma vez que o comportamento alimentar se relaciona com a subjetividade, muitas vezes não acessível ao profissional de saúde.

\section{Conclusão}

Faz-se necessário maior incentivo aos bons hábitos alimentares para os universitários avaliados, incluindo a procura do profissional nutricionista para esclarecer dúvidas pertinentes ao cotidiano alimentar, porém é necessário o entendimento subjetivo relacionado às escolhas alimentares. É imprescindível a formação de pedagogos aptos para defender a ideia de que o tema da alimentação e nutrição é fundamental no projeto político-pedagógico da escola. Aliar a educação nutricional com a prática pedagógica consciente dos profissionais capacitados pode ser uma alternativa para a real mudança dos hábitos alimentares.

\section{Referências}

1. World Health Organization. Diet, nutrition and the prevention of chronic diseases. Report of a Joint FAO/WHO/UNU Expert Consultation. Geneva: World Health Organization; 2003.

2. World Health Organization. Reducing risks, promoting healthy life. World Health Report. Geneva: World Health Organization; 2002.

3. Souza JA. Conhecimentos nutricionais: reprodução e validade de questionário. Dissertação [Mestrado em Saúde] - Universidade do Porto; 2009.

4. Seabra KC, Moura MLS. Alimentação no ambiente de creche como contexto de interação nos primeiros dois anos de um bebê. Psicol Estud 2005;10(1):77-86.

5. Rossetti-Ferreira MCA. Necessária associação entre o educar e cuidar. Pátio Edeuc Infant 2003;1(1):10-2.

6. Veiga IPA. Projeto político-pedagógico da escola: uma construção coletiva. In: Veiga IPA. Projeto políticopedagógico da escola: uma construção possível. Campinas: Papirus; 2007. p.11-35

7. Bourdieu, P. Razões práticas: sobre a teoria da ação. Campinas: Papirus; 2004.

8. Marinho MCS, Hamann EM, Lima ACCS. Práticas e mudanças no comportamento alimentar na população de Brasília, Distrito Federal, Brasil. Rev Bras Saúde Mat Infant 2007;7(3):251-61.
9. Dias SLA, Sieben M, Cozer P, Alves RB, Halbert T. Estatuto da Criança e do Adolescente: aprendendo cidadania. Inclusão Social 2007;2(2):116-23.

10. Kuczmarski MF, Kuczmarski RJ, Najjar M. Effects of age on validity of self-reported height weight, and body mass index: findings from of Third National Health and Nutrition Examination Survey, 1988-1994. J Am Diet Assoc 2001;101:28-34.

11. World Health Organization. Physical status: the use and interpretation of anthropometry. Geneva: World Helth Organization; 1995.

12. Fisberg RM, Slater B, Marchioni DML, Martini LA. Inquéritos Alimentares: Métodos e bases científicas. Barueri: Manole; 2005.

13. Guadagnin SC. Elaboração e validação de questionário de conhecimentos em nutrição para adultos. Dissertação [Mestrado em Nutrição Humana] - Faculdade de Ciências da Saúde. Universidade Nacional de Brasília; 2010.

14. Casarin RCV, Fernandes DRM, Lima-Arsati YBO, Cury JA. Concentração de fluoreto em arroz, feijão e alimentos infantis industrializados. Rev Saúde Pública 2007;41(4):549-56.

15. Dalla Costa MC, Cordini Jr L, Matsuo T. Hábito alimentar de escolares adolescentes de um município do oeste do Paraná. Rev Nutr 2007;20(5):461-71.

16. Rossi A, Moreira EAM, Ruen MS. Determinantes do comportamento alimentar: uma revisão com enfoque na família. Rev Nutr 2008;21(6):739-48.

17. Pesquisa de orçamentos familiares 2008-2009: antropometria e estado nutricional de crianças, adolescentes e adultos no Brasil / IBGE, Coordenação de Trabalho e Rendimento. Rio de Janeiro: IBGE; 2010.

18. Batista Filho M, Rissin A. A transição nutricional no Brasil: tendências regionais e temporais. Cad Saúde Pública 2003;19(1):181-91.

19. Silva ACA, Telarolli Jr R, Monteiro, MI. Analisando conhecimentos e práticas de agentes educacionais e professoras relacionados à alimentação infantil. Ciênc Educ 2010;16(1):199-214.

20. Silva JG, Teixeira MLO, Ferreira MA. Alimentação e saúde: sentidos atribuídos por adolescentes. Esc Anna Nery 2012;16(1):88-95.

21. Sichieri R, Coitinho DC, Monteiro JB, Coutinho WF. Recomendações de alimentação e nutrição saudável para a população brasileira. Arq Bras Endocrinol Metab 2000;44(3):227-32.

22. Bigio RS, Verly Junior E, Castro MA, César CLG, Fisberg RM, Marchion DML. Determinantes do consumo de frutas e hortaliças em adolescentes por regressão quantílica. Rev Saúde Pública 2011;45(3):448-56.

23. Ramalho AA, Dalamaria T, Souza OF. Consumo regular de frutas e hortaliças por estudantes universitários em Rio Branco, Acre, Brasil: prevalência e fatores associados. Cad Saúde Pública 2012;28(7):1405-13.

24. Monteiro CA, Mondini L, Costa RBL. Mudanças na composição e adequação nutricional da dieta familiar nas áreas metropolitanas do Brasil (1988-1996). Rev Saúde Pública 2000;34(3):251-8.

25. Monteiro CA, Benicio MHDA, Freitas ICM. Melhoria em indicadores da saúde associados à pobreza no Brasil dos anos 90: descrição, causas e impacto sobre desigualdades regionais. São Paulo: NUPENS/USP; 1997.

26. Davanco GM, Taddei JAAC, Gaglianone CP. Conhecimentos, 
atitudes e práticas de professores de ciclo básico, expostos e não expostos a Curso de Educação Nutricional. Rev Nutr 2004; 17(2):177-84.
27. Oliveira SP, Thébaud-Mony A. Estudo do consumo alimentar: em busca de uma abordagem multidisciplinar. Rev Saúde Pública 1997;31(2):201-8. 